\title{
Concealment Stability Based Path Clout Expedient In Brume-Based Utility
}

\author{
Sangu Raja Reddy, M.Naresh
}

\begin{abstract}
With the quick increase of computer innovation, cloud-primarily based truely answers have clearly turn out to be a heat situation be counted. They now not absolutely supply clients with advantage, regardless of the truth that additionally convey severa safety troubles, like data sharing and also personal privacy challenge. At a few diploma on this paper, we have a tendency to provide Partner in Nursing advantage get proper of access to to govern tool with opportunity splitting up based totally completely upon privacy safety (PS-ACS). In the PS-ACS style, we regularly have a tendency to break up customers right into private place name (PRD) and building proper (PUD)realistically. In PRD, to accumulate browse benefit get right of access to to permission and moreover write access authorization, we undertake the KeyAggregate coding (KAE) and the Improved Attribute-primarily based definitely definitely Trademark (IABS) severally. In PUD, we regularly have a tendency to accumulate new multi-authority ciphertext insurance characteristic-based completely surely coding $(C P-A B E)$ scheme with cheaper decipherment to avoid the troubles of single reason of failure further to ultra-modern crucial distribution, similarly to fashion Associate in Nursing price-effective feature retraction technique for it. The evaluation and furthermore simulation cease end result show that our trouble is viable and superior to secure clients' personal privacy in cloud-primarily based totally totally offerings.
\end{abstract}

\section{INTRODUCTION}

With the short enhancement of dispensed computing, awesome records and open cloud managements had been generally used. Clients can save their information inside the cloud gain and additionally rely on the cloud advantage issuer to offer records accessibility to unique customers. All the same, the cloud expert co-op can't be virtually trusted. Because it may deliver records get proper of entry to to three illicit customers or assailants income driven benefit. For clients, it is vital to take complete amazing setting of dispensed garage vicinity benefit, and moreover moreover to assure facts security. In this manner, the studies of benefit get proper of access to to govern plan to make sure customers' safety and safety in cloud hassle is of exquisite essentialness. Considering that everyday get admission to control technique [1] can't sufficiently fathom the protection worries that exist in data sharing, one-of-a-kind plans to collect safety in addition to unscrambling of statistics sharing have clearly been proposed. In 2007, Bethen court docket docket et al. [2] first proposed the ciphertext technique building based honestly encryption (CP-ABE). Regardless of, this plan does not consider the rejection of get right of entry to approvals. Attrapadung et al. [3, 4] idea of consumer revocable $\mathrm{ABE}$ conspire. All the equal, they will be now not great within the re-appropriating trouble. In
2011, Hur et al. [5] set onward a great grained denial conspire, however the reality that it may without an entire lot of a stretch reason key escrow scenario. Lewko et al. [6] used multi-expert ABE ( MA-ABE) to make smooth critical escrow issue. However, the entryway technique isn't bendy. Later, Li et al. [7] added an facts sharing plot primarily based absolutely mostly on vital feature file encryption, which blesses extraordinary accessibility authorizations to Information safety problems added with the beneficial useful resource of records sharing have without a doubt extensively hindered the occasion of cloud computing, severa solutions to gain mystery writing similarly to coding of facts sharing are prepared. But it's miles no longer reasonably-priced from the exquisite further to effectiveness. Chen et al. Deliberate Key-Aggregate secret writing guiding principle, well lowering the size of the ciphertext further to a quit end result the call of the sport, despite the reality that simplest for subjects any location the records owner recognizes the client's identification. We advocate an specific accessibility device added up as PSACS, that is privilege splitting up sustained privacy protection. The device uses Key-Aggregate cryptography (KAE) problem depend similarly to Pecking order Attributebased totally cryptography (HABE) motif to carry out browse accessibility management subject matter within the PSD and additionally pudding severally. The KAE motif extensively boosts accessibility effectiveness and moreover however furthermore the HABE fashion in particular lowers the challenge of one authority similarly to shields the nonpublic privateness of customer records. Compared to the MAH-ABE style that does not sit down with the write benefit get right of get right of access to toto govern, we've got absolutely received a curved to utilize degree Enhanced Attribute-based completely Trademark (IABS) style to impose compose accessibility control in the PSD. Throughout this style, the patron can pass the cloud server's signature confirmation even as no longer disclosing the identification, similarly to properly change the document.

\section{RELATED WORK}

Before revealing our organized for secure take a look at custom designed, we have a tendency to regularly will be inclined to in the starting collect accomplice diploma effect

Revised Manuscript Received on September 10, 2019.

Sangu Raja Reddy *, CSE department, Newtons Institute of Engineering, Macherla, A.P. India.

M.Naresh, CSE department, Newtons Institute of Engineering, Macherla, A.P. India. 
for the documentations performed in the later, every considered absolutely one in every of Which are recorded in Table one. The PRD has series of clients, and moreover their Personalities are tremendous to the bourgeois. At the reasoning at the same time as all is adhered in carried out, the statistics consultant most effective dreams the clients to trigger to or as an alternative on the in evaluation hand correction segments of facts fi les, and unequivocal customers can gather and moreover exchange unique segments of the information. For example, the weblog owner can empower his buddy to preserve in mind some small uncertain quantity of his private pics; undertakings can in like methods finance reps to accumulate or revision a few little unsure amount of fragile knowledge. This name for the statistics expert to permit customers sees or makes see the possibility to alter to a tough and rapid of information. In Chen's [15] MAH-ABE extend, the CP-ABE is accomplished to accomplish the see get to endorsement, but there are multiple disfigurements to be idea of. Straightaway, considering that during PRD, every purchaser consists of organization with the representative and furthermore furthermore the range is actually worrying now not some thing, there can be no name for to apply the $\mathrm{CP}$ $\mathrm{ABE}$ this is suitable to the problem that consists of accomplice quantity of customers, similarly to their personalities are dull to the bourgeois, at the equal time because the KAE tale awaits the small customers with excellent characters. Furthermore, the dissipating in addition to the primary desire of keys in addition to top functions, safety moreover, system of CP-ABE get on a definitely vital diploma in addition extraordinary separated similarly to furthermore the KAE story. Within the center of this manner, the KAE plot is gotten to perform the see get to endorsement that revamps the section effectiveness. In context of the pinnacle than assessment, the paper makes use of the Key-Aggregate mystery developing assignment to decide the records facts to famend great test notification the opportunity to administrate After that the owner's purchaser software program software runs Encrypt of KAE the use of most of the people key and the shape of class information to encrypt the PHR documents and sends them to the cloud. Gain get entry to to and moreover essential motion. When the character sends out accessibility name for to the cloud server, and his file index quantity is, after that the cloud server returns the matching encrypted kind file to the purchaser. Generate an gathered decryption thriller for a set of ciphertext training using Extract of KAE further to despatched it to the identical character, Finally, any sort of patron with an aggregate mystery can decrypt any shape of ciphertext whose elegance is blanketed within the mixture key via Decrypt of KAE.

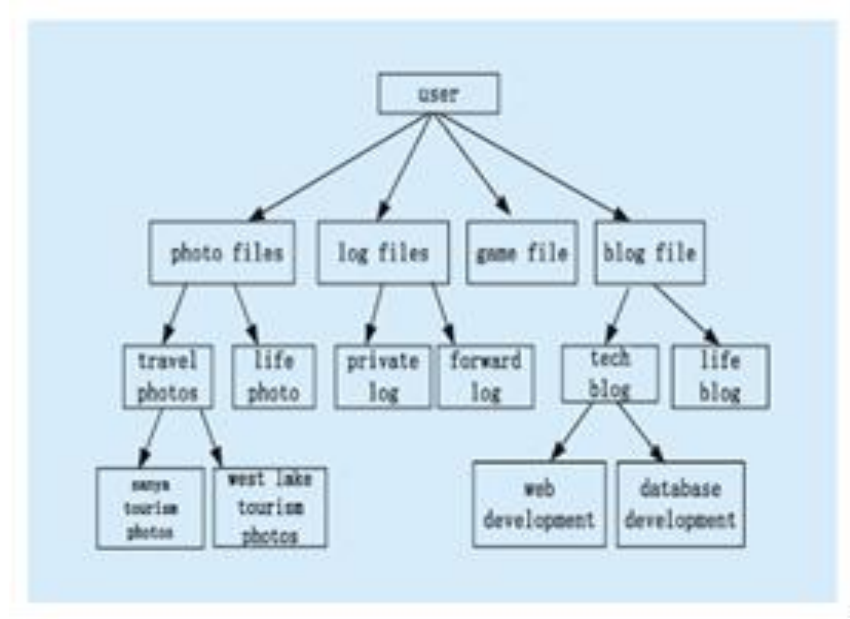

3. Encrypt (PP, PK, I, M). On enter the majority parameter $\mathrm{PP}$, the general public vital $\mathrm{PK}$, the file index variety $i$, the facts files $m$ in addition to a random quantity, the information owner computes the ciphertextasand after that sends out the ciphertext to the cloud net server.

Remove (MS K, S). The Remove additives is achieved with the aid of the use of the authority. On enter the draw close male thriller critical MSK and furthermore a hard and speedy $\mathrm{S}$ of the document index massive variety which can be permitted the benefit get proper of access to to authorization via the use of manner of the data proprietor. It outputs the gathered thriller as

$$
C T=C_{1}=\mathrm{g}^{2}, C_{2}=\left(\mathrm{g}^{3} \mathrm{~g}_{1}\right)^{2}, C_{3}=\mathrm{e}\left(\mathrm{g}_{1} \mathrm{~g}^{\mathrm{n}}\right)^{1}
$$

Decrypt (K, S, I, CT ). The decryption additives takes as enter the buildup thriller, the collection $\mathrm{S}$, the report index variety $i$ and additionally the ciphertext $C T$. It recuperates $m$ as.

$$
\begin{aligned}
& K_{\delta}=\prod_{j \in s} g_{n+1-j}^{\beta} \\
& m=C_{3} \cdot \mathrm{e}\left(\mathrm{K}_{\delta} \cdot \prod_{\mathrm{j} \in \mathrm{s}, \mathrm{j}+1} \mathrm{~g}_{\mathrm{n}+1-\mathrm{j}+1}, C_{1}\right) \\
& / \mathrm{e}\left(\prod_{\mathrm{j} \in \mathrm{S}} \mathrm{gg}_{\mathrm{n}+1-\mathrm{j}}, C_{2}\right)
\end{aligned}
$$

As Chen's MAH-ABE plan does now not speak over with the write get right of access to control, and additionally in PRD some instances exist, as an example, the owner requires his particular pals to adjust his information after he reviewed it. So we proposed the create gain access to permission in PRD. For the consumer, the general public trick and records elegance tag are all understood, he can use the set of guidelines to encrypt the files after he changed, and afterwards placed up them to the cloud. However whether or not or not or not the cloud internet server 
conserves the modified files is determined with the useful resource of the compose accessibility manipulate insurance. On the handiest hand, within the complicated cloud surroundings, if a consumer's adjustment operations are very ordinary, possibly he may be very critical to the man or woman, to make certain that the purchaser may be troubled from outdoor assaults. For that motive, the person issues the leakage of identity after the trademark. On the alternative hand, within the facts sharing plan, the separate get entry to of test and call the report may be very essential. In PRD, not all customers that have in reality checked out approvals likewise have compose permissions to the files. Whether the consumer has create approvals to the file is determined with the aid of manner of the information proprietor. For that purpose, this paper selects the progressed feature-primarily based absolutely actually trademark (IABS) to determine out the person's write permission.

\section{EXPERIMENTAL REVIEW \& RESULTS}

The PUD is defined via a huge shape of customers, a notable deal of attributes possessed through the man or woman, complexity control, and uncertain human beings' identity. Because the above developments, the character can actually have the have a look at advantage get right of get entry to toto approval. Although the function-primarily based absolutely absolutely report encryption gadget (CP$\mathrm{ABE}$ ) can achieve gain get proper of access to to govern, it cannot fulfill the desires of complex cloud environment. In elegant $\mathrm{CP}-\mathrm{ABE}$ device, there may be simply one feature authority accountable for the management of tendencies further to distribution of keys.

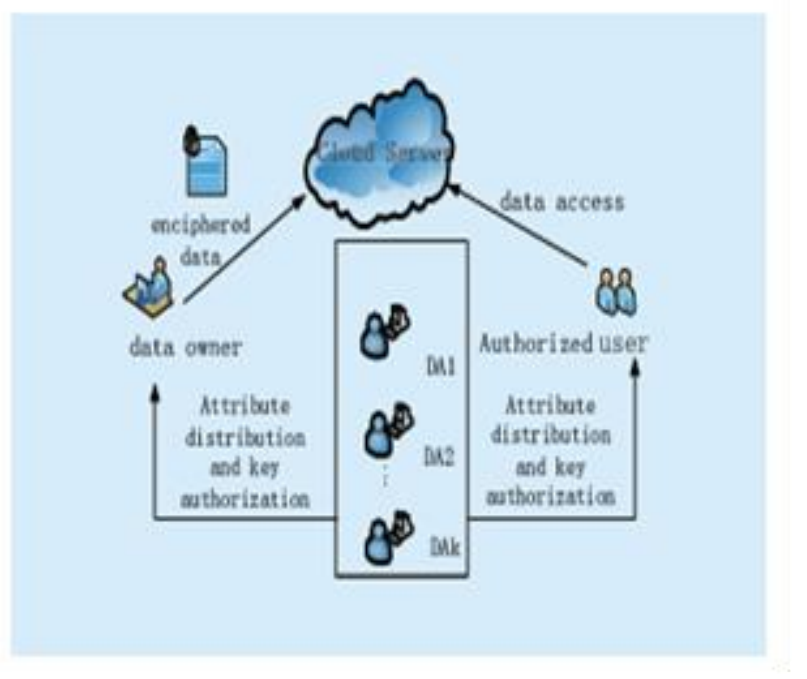

The authority is probably a university registrar's place of job, the organisation's Human Resources department or authorities educational organizations and rapid. The facts owner specifies get proper of access to suggestions in addition to secures the records documents primarily based completely completely mostly on this plan. Each individual is dispersed a essential associated with his characteristic. As extended as the character's developments fulfill the benefit get admission to to coverage he can decrypt the record. Nevertheless, if there can be most effective one authority inside the device and all public and moreover private tips are released through the authority. 2 problems will in truth show up within the beneficial software program software: 1) In the practical cloud placing, there are a amazing deal of government and moreover every authority of their very nonpublic region appears after part of clients' traits. The attributes owned with the resource of the man or woman are supplied from numerous authorities encryption set of guidelines takes as inputs most people specs PP, most of the people important PK, the appropriate public super tricks, the message $\mathrm{m}$ and an LSSS get entry to shape over all the chosen attributes from the entailed government

$$
\text { . } C T=
$$

$$
\left(\begin{array}{c}
C=m \cdot e\left(g_{1}, g_{2}\right)^{\propto 1}, C^{1}=g_{1}^{2} \\
\left.C_{1}=g_{2}^{1 \lambda} \cdot\left(g_{2}^{I D} \cdot H(\rho(i))\right)^{y i}, D_{i}=g_{1}^{n}\right)_{j \in(i \ldots \ldots n}(i \ldots . . .
\end{array}\right)
$$

Trick Gen (PP, PK, MS K, S ). Each authority runs the important era technique. On enter most people parameter PP, the applicable public function keys PK, the draw close thriller essential MSK and moreover a set of features dealt with thru the authority $S$, it outputs the exchange secrets and techniques as

$$
\begin{array}{r}
T K=\left\{k=g_{2}^{\frac{\alpha}{t}} g_{2}^{\frac{\alpha \beta}{t}}, L=g_{1}^{\beta t}, \forall_{x \in S} k_{\delta}\right. \\
\left.=\left(g_{2}^{I D} \cdot H(\rho(i))\right)^{\beta / t}\right\}
\end{array}
$$

in which is a random big range bind with every customer and permit because the man or woman's non-public key.Transform (TK, CT ). The alternate technique takes as input the transformation crucial TK and a cipher text CT. If $\mathrm{S}$ does now not satisfy the gain get proper of access to $e\left(C^{1}, K\right) / \prod_{i \in I}\left(e\left(c_{i}^{w 0}, L\right) \cdot\left(e\left(D_{i}^{w 0}, K\right)=\right.\right.$ $e\left(g_{1}, g_{2}\right)$

it chooses a difficult and rapid of constants, such that if stand stocks of the name of the game s in step with $\mathrm{M}$, after that, in which

. After that the cloud net server computes and sends the

Decrypt (). The decryption formula takes as enter the in detail decrypted ciphertext and moreover an person private key SK. It calculates

$$
m=C / T, T=e\left(g_{1} g_{2}\right)^{\frac{\alpha \beta}{t}}
$$

Notification that considering that the ciphertext is already in part decrypted thru using the cloud internet server, the consumer certainly calls for one exponentiation gadget to recoup the message.

TKUpdate (TK, TUK). Upon getting the makeover replace essential, the cloud server runs the improvement critical decorate method to enhance the identical makeover keys in terms of each non-revoked man or woman that has

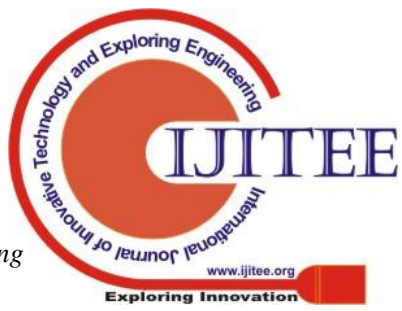


the feature. Hence the trade essential TK may be upgraded as

$$
T K^{1}=\left(\begin{array}{c}
\left.K=g_{2}^{\frac{\alpha}{t}} g_{2}^{\frac{\alpha \beta}{t}}, L=g_{1}^{\frac{\beta}{t}}\right\} \\
K_{X \emptyset I}=\left(g_{2}^{I D} \cdot H(x)\right)^{\frac{\beta}{t}} \\
k_{x=t}^{*}=\left(g_{2}^{I D}, H(x)\right)^{\frac{\beta}{t}}
\end{array}\right)
$$

Upon receiving the ciphertext beautify key, the cloud server runs the ciphertext re-encryption set of policies to enhance the equal ciphertext.

\section{CONCLUSION}

In this paper, we endorsed an entryway control form (PSACS), it's miles gain dividing in view of protection assure. Through the exam of cloud scenario and the pinnacle inclinations of the purchaser, we dividers customers into man or woman region (PRD) and open region (PUD) appropriately. In PRD, we set up test in addition to compose get to has the equal opinion for clients in my view. To reap take a look at out acquire consent, the KAE conspire that could enhance the entryway efficiency is acquired. A excessive diploma of consumer protection is assured at the exact equal time with the useful resource of way of the use of IABS conspire which could make a preference clients' make up gain consent. For customers in PUD, we built one extra multi-expert ciphertext technique specific based totally totally truly encryption (CP-ABE) conspire with efficient unscrambling to live away from the issues of unmarried reason of sadness and furthermore pressured crucial appropriation, and plan an efficient unique disavowal technique for it. The assessment furthermore, the pastime cease result display that the PSACS tale is practical and better than ensure the safety of statistics in cloud-based totally truely managements.

\section{REFERENCES}

1. YU SH, WANG C, REN K, "Achieving Secure, Scalable, and also Fine-Grained Data Gain Access To Control in Cloud Computing", Procedures of IEEE Disadvantage- ference on Details Communications 2010, pp. 1-9, 2010.

2. BETHENCOURT J, SAHAI A, WATERS B, "Ciphertext-Policy Attribute-based File Encryption", IEEE Seminar on Security and also Personal privacy, vol. 2008, no. 4, pp. 321-334, 2007.

3. ATTRAPADUNG N, IMAI H, "Conjunctive Program as well as Attribute-Based File Encryption", Process of Pairing-based Cryptography-- Coupling 2009, vol. 5671, pp. 248-265, 2009.

4. ATTRAPADUNG N, IMAI H, "Attribute-Based File Encryption Sustaining Direct/Indirect Revocation Modes", Proceedings of Cryptography as well as Coding 2009, pp. 278-300, 2009.

5. HUR J, NOH D K, "Attribute-based Accessibility Control with Efficient Revocation in Information OutsourcingSystems", IEEE Purchases on Identical andDistributedEquipments, vol. 22, no. 7, pp. 1214-1221, 2011.

6. LEWKO A, SEAS B, "Decentralizing Quality- based Security", Procedures of Breakthroughs in CryptologyEUROCRYPT 2011 - 30th Annual International Seminar on the Concept and also Applications of Cryptographic Techniques, pp. 568-588, 2011.
7. LI M, YU SH, ZHENG Y, "Scalable as well as Secure Sharing of Personal Wellness Records in CloudComputing Utilizing Attribute-based Encryption", IEEE Deals on Parallel and also DistributedSystem, vol. 24, no. 1, pp. 131-143, 2013.

8. XIE X, MA H, LI J, et al, "New Ciphertext-Policy Attribute-based Access Control with Effective Retraction", Proceedings of Info and Communication Modern technology 2013, pp. 373-382,2013.

9. LIANG K, MAN H A, SUSILO W, et alia, "An Adaptively CCA-Secure Ciphertext-Policy AttributeBased Proxy Re-Encryption for Cloud Information Sharing", Information Safety Method and alsoExperience, pp. 448-461, 2014.

10. CHU C K, CHOW S M, TZENG W G, "Key-Aggregate Cryptosystem for Scalable Information Cooperating Cloud Storage Space", IEEE Transactions on Parallel and Dispersed Equipments, vol. 25, no. 2, pp. 468-477, 2014.

11. LI J, KIM K, "Concealed Attribute-based Trademarks without Anonymity Retraction", Information Sciences, vol. 180, no. 9, pp. 1681-1689, 2010.

12. MAJI H K, PRABHAKARAN M, ROSULEK $M$, "Attribute-based Trademarks", Procedures of RSA Conference 2011, pp. 376-392, 2011.

13. KUMAR S, AGRAWAL S, BALARAMAN S, et alia, "Connect based Trademarks for BoundedMulti-level Threshold Circuits", Procedures of Public Key Infrastructures, Services as well as ApplicationsEuropean Workshop, Europki 2010, pp.141-154, 2010.

14. BEIMEL A, "Protect Systems for Secret Sharing and Secret Circulation", International Journal of Pure \& Applied Math, Research Study Thesis,1996.

15. CHEN D, SHAO J, FAN X, "MAH-ABE based Privacy Access Control in Cloud Computing", Chinese Journal of Electronic devices, vol. 42, no. 4, pp.821-827, 2014.

16. NARAYAN S, GAGNÉ M, SAFAVI-NAINI R, "Privacy Preserving EHR System Using Attribute-based Infrastructure", Process of ACM Cloud Computer Safety Workshop 2010, pp.47-52, 2010.

17. RUJ S, NAYAK A, STOJMENOVIC I, "DACC: Dispersed Accessibility Control in Clouds", Proceedings of Count On, Security and Privacy in Computing as well as Communications 2011, pp. 91-98, 2011.

18. AKINYELE $\mathrm{J}$ A, ENVIRONMENT-FRIENDLY M RUBIN A D, "Charm: A Structure for Quickly Prototyping Cryptosystems", Journal of Cryptographic Design, vol.3, no. 2, pp. 111-128, 2011.

19. LYNN B, "The Stanford Pairing based Crypto Collection", http://crypto.stanford.edu/pbc.

20. Appeal, http://www.charm-crypto.com. 